\title{
COVID-19 Pandemic in India: What Lies Ahead
}

\author{
Prasenjit Mitra $^{1} \cdot$ Sanjeev Misra ${ }^{2} \cdot$ Praveen Sharma $^{1}$
}

Published online: 20 April 2020

(C) Association of Clinical Biochemists of India 2020

The first case of coronavirus disease presented with pneumonia of unknown cause in the town of Wuhan, China. It was reported to the Country office of WHO, China on December 31, 2019. Since then, the disease had rapidly spread to the rest of the World accounting for high morbidity and mortality. The disease was announced a Public Health Emergency of International Concern on January 30, 2020. On February 11, 2020, the WHO declared COVID-19 as a new name for the disease, while the International virus classification commission called the novel coronavirus as severe acute respiratory syndrome coronavirus 2 (SARS-CoV-2). The disease continued to spread in such a large proportion affecting several countries that WHO characterised it as a pandemic on March 11, 2020. As on April 04, 2020, around 972,303 diagnosed cases of COVID-19 with 50,322 deaths and 154,430 recoveries have been reported [1]. Globally, United States of America (USA), Italy, Spain, China, Germany, Iran have been the most affected countries. In India, the cases of COVID-19 have increased alarmingly in the last 7 days. On March 28, 2020, the total confirmed COVID-19 cases were 775 with 19 deaths. As on April 04, 2020, this number has increased to 3127 confirmed cases and 86 deaths [2].

The SARS-CoV-2 is a novel member of coronavirus family. Coronavirus are enveloped positive sense single strand RNA viruses (genome size 36-42 kb, diameter: 60-140 nm) having surface projections resembling a crown

Praveen Sharma

praveensharma55@gmail.com

1 Department of Biochemistry, All India Institute of Medical Sciences, Jodhpur, India

2 Department of Surgical Oncology, All India Institute of Medical Sciences, Jodhpur, India under the electron microscope. This crown like appearance is the basis behind the name, Coronavirus. These viruses are zoonotic pathogens; they originate in wild animals and spill over from wildlife into humans causing disease. In the past 20 years, two members of the same family, the SARS$\mathrm{CoV}$ and MERS-CoV have caused epidemic outbreaks of Severe Acute Respiratory Syndrome (SARS) and Middle East Respiratory Syndrome (MERS) respectively. SARSCov-2 shares $88 \%$ genetic similarity with SARS-CoV and $50 \%$ similarity with MERS-CoV [3].

During the last 4 months, several studies have been published on COVID-19 ranging from case-reports, caseseries and large population studies that have reported the clinical features, laboratory findings and diagnostic evaluation of individuals suffering from this disease. The major cause of death is reported to be adult respiratory distress syndrome (ARDS) with occasional cases of multiorgan failure. Advancement in diagnostics via nucleic acid testing played a major role in detecting the sequences of viral genome. But there is probability of some degree of misdetection owing to the over-hypersensitivity and sampling dependence. As the virus continues its wrath, the major concern is still the unavailability of any cure. Only supportive treatment can be administered, thereby urging the immediate need for the development of effective preventive and therapeutic strategies.

Rodriguez-Morales et al. [4] analysed 19 different studies in a meta-analysis and reported that fever, cough and dyspnoea were the most common manifestations among the 656 COVID-19 patients. Around 32.8\% developed ARDS, $20.3 \%$ patients required critical care, and $6.2 \%$ developed shock. Fatal outcomes were observed in $13.9 \%$ patients. Old age was significantly associated with the severity of the disease [4]. Similar clinical manifestations were observed in another meta-analysis by Yang et al. 
which included eight studies with 46,248 infected patients and reported that hypertension, diseases of respiratory and cardiovascular system may be significant risk factors for severity of COVID-19 [5]. Later, these findings were supported by Emami et al. in another meta-analysis. After systematically analysing the data of 76,993 patients, smoking history and diabetes was also added to the list [6].

Only a small proportion of the infected patients progress to severe stage needing critical care. Thus, in absence of proper cure, it becomes essential to delineate the factors that may aid in the severity assessment of the disease. If individuals at risk of developing severe symptoms can be identified early, management of the disease may improve to a significant extent. In this context, several studies have tried to establish the laboratory abnormalities that may aid in discriminating severe and non-severe cases. Among the laboratory parameters, Guang et al. reported increased alanine transaminase (ALT), lactate dehydrogenase (LDH), D-dimer, C-Reactive Protein, ferritin along with significantly raised interleukins (IL-2R, IL-10, IL-6 and TNF- $\alpha$ ) in severe cases of COVID-19 in comparison to non-severe cases. A significantly lower absolute number of lymphocytes, $\mathrm{CD} 4^{+} \mathrm{T}$ and $\mathrm{CD} 8^{+} \mathrm{T}$ cells along with downregulation of IFN- $\gamma$ expression in severe cases was also observed [7]. In another pre-print, Xiang et al. reported serum urea, creatinine and cystatin $\mathrm{C}$ may be potential biomarkers in severity of COVID-19 [8]. Huang et al. reported higher plasma levels of interleukins (IL-2, IL-7, IL-10) and other inflammatory markers (GSCF, IP10, MCP1, MIP1A and TNF- $\alpha$ ) in COVID-19 patients requiring ICU care versus non-ICU patients [9].

Since the virus shares genetic similarity with SARS$\mathrm{CoV}$ and MERS-CoV, researchers are expecting a similar molecular pathogenesis. Previous studies have shown the occurrence of a cytokine storm in SARS and MERS. Cytokine storm syndrome is a state of sudden surge of proinflammatory cytokines due to stimulation by drugs or pathogens that disrupts the normal balance of pro- and antiinflammatory cytokines. Upon infection, the virus may abnormally activate various immune cells like dendritic cells, lymphocytes, natural killer cells and macrophages. Once activated, these cells produce high amounts of proinflammatory cytokines which further involves more immune cells leading to a positive feedback cycle. Upon crossing a certain threshold, this may lead to a cytokine storm characterised by aggravation of clinical symptoms along with diffuse intravascular coagulation, shock, multiorgan failure and death. It is now strongly believed that cytokine storm may lead to the transition from mild to severe in COVID-19 patients [10]. If this link can be established, then existing interleukin blockers may be used for management of severe COVID-19 patients.
There are several research questions still unanswered in relation to COVID-19. One of our interest is alternative diagnostic modalities. As of now, real time PCR based SARS-CoV-2 RNA detection from respiratory samples is the specific diagnostic test of COVID-19. But there are issues with sampling and other technicalities in this test. Recently, ELISA kits based on detection of IgM and $\operatorname{IgG}$ antibodies against $\mathrm{N}$ protein of SARS-CoV-2 have been made available [11]. This has opened the possibility of diagnosing an ongoing as well as past infection. But there is a strong need to provide newer ELISA kits to detect viral antigens that may provide an alternative to viral RNA detection.

Further, most of the research data in relation to COVID19 has been obtained from studies in Chinese population. With the recent surge of infections in USA, Italy, Spain and other countries, data from these populations would add significant knowledge in understanding this disease. Thus, prospective studies should be planned to evaluate the pathogenesis, viral dynamics, the clinical and laboratory spectrum of the disease and to identify mechanistic changes that may aid in therapeutic strategies either by novel cure or by drug repurposing.

COVID-19 has been the biggest public health crisis state since World War II. It has not limited itself as a medical emergency; it is affecting the global economy and if proper measures are not taken, it could have serious implications in socio-economic status and daily lives of mankind. It has deeply challenged the health care infrastructure especially in low- and middle-income countries. Every country is trying to protect its populations and the health workers as effectively as possible. India has already implemented nationwide lockdown since March 25, 2020 with the vision to 'break the chain' of infection and control its transmission. As the virus threatens community spread, there is an urgent need to improve infrastructure, develop novel testing facilities, prepare human resources, support frontline health workers and search for a cure until the battle is won.

\section{References}

1. World Health Organization. Coronavirus disease 2019 (COVID19) situation report-74. https://www.who.int/emergencies/dis eases/novel-coronavirus-2019/situation-reports/. Accessed 4 Apr 2020.

2. COVID-19 Tracker I India [Internet]. Covid19india.org. 2020. https://www.covid19india.org/.

3. Li X, Geng M, Peng Y, Meng L, Lu S. Molecular immune pathogenesis and diagnosis of COVID-19. J Pharm Anal. 2020. https://doi.org/10.1016/j.jpha.2020.03.001.

4. Rodriguez-Morales A, Cardona-Ospina J, Gutiérrez-Ocampo E, Villamizar-Peña R, Holguin-Rivera Y, Escalera-Antezana J, et al. Clinical, laboratory and imaging features of COVID-19: a 
systematic review and meta-analysis. Travel Med Infect Dis. 2020. https://doi.org/10.1016/j.tmaid.2020.101623.

5. Yang J, Zheng Y, Gou X, Pu K, Chen Z, Guo Q, et al. Prevalence of comorbidities in the novel Wuhan coronavirus (COVID-19) infection: a systematic review and meta-analysis. Int J Infect Dis. 2020. https://doi.org/10.1016/j.ijid.2020.03.017.

6. Emami A, Javanmardi F, Pirbonyeh N, Akbari A. Prevalence of underlying diseases in hospitalized patients with COVID-19: a systematic review and meta-analysis. Arch Acad Emerg Med. 2020;8(1):e35.

7. Chen G, Wu D, Guo W, Cao Y, Huang D, Wang H, et al. Clinical and immunological features of severe and moderate coronavirus disease 2019. J Clin Invest. 2020. https://doi.org/10.1172/ JCI137244.

8. Xiang J, Wen J, Yuan X, Xiong S, Zhou X, Liu C, et al. Potential biochemical markers to identify severe cases among COVID-19 patients. medRxiv. 2020. https://doi.org/10.1101/2020.03.19. 20034447.
9. Huang C, Wang Y, Li X, Ren L, Zhao J, Hu Y, et al. Clinical features of patients infected with 2019 novel coronavirus in Wuhan, China. Lancet. 2020;395(10223):497-506.

10. Chen C, Zhang XR, Ju ZY, He WF. Advances in the Research of Cytokine Storm Mechanism Induced by Corona Virus Disease 2019 and the Corresponding Immunotherapies. Zhonghua Shao Shang Za Zhi. 2020;36:E005. https://doi.org/10.3760/cma.j. cn501120-20200224-00088.

11. Yuen K-S, Ye Z-W, Fung S-Y, Chan C-P, Jin D-Y. SARS-CoV-2 and COVID-19: the most important research questions. Cell Biosci. 2020;10(1):40. https://doi.org/10.1186/s13578-020-004044.

Publisher's Note Springer Nature remains neutral with regard to jurisdictional claims in published maps and institutional affiliations. 